\title{
Erythroid Transcription Factor NF-E2 Coordinates Hemoglobin Synthesis ${ }^{1}$
}

\author{
NANCY C. ANDREWS \\ Division of Hematology-Oncology, Children's Hospital, Department of Pediatrics, Harvard \\ Medical School, Howard Hughes Medical Institute, Boston, Massachusetts 02115
}

Red blood cells have a remarkably simple existence. Fully differentiated erythrocytes are tiny, anucleate sacs of $\mathrm{Hb}$ that deliver oxygen to all tissues of the body. Although each erythrocyte circulates for about $4 \mathrm{mo}$, basal renewal of the hemoglobinized red cell mass requires daily production of more than $6 \mathrm{~g}$ of $\mathrm{Hb}$ in an average adult. This extraordinary synthetic feat is unparalleled in other tissues.

$\mathrm{Hb}$ is among the most intensively studied of all eukaryotic proteins. It is a complex molecule, made up of two $\alpha$-globin chains and two $\beta$-globin chains, supporting four iron-laden heme groups. Assembly of $\mathrm{Hb}$ requires multistep biosynthesis of protoporphyrin, incorporation of elemental iron to form heme, and production of $\alpha$ - and $\beta$-globin proteins. There is ample clinical evidence that these processes must be carefully coordinated to avoid the toxicity of unused components.

Imbalanced globin chain synthesis results in thalassemia, a condition affecting millions of individuals worldwide. Lesions in the heme biosynthetic pathway lead to accumulation of unused iron and chemical intermediates, resulting in porphyrias and sideroblastic anemias. And even subtle excesses in iron uptake may result in iron overload and damage to a variety of tissues, as seen in hereditary hemochromatosis.

We would like to understand how the multiple steps leading to $\mathrm{Hb}$ synthesis are regulated and coordinated, as a paradigm for understanding molecular events in cellular determination and differentiation. We believe that insights into this process will aid in designing novel therapies to treat hematologic diseases. I will first give a brief overview of erythrocyte gene expression, focusing on the globin genes, and then describe a unique transcriptional regulatory factor that appears to coordinate all three aspects of $\mathrm{Hb}$ formation-globin protein production, iron uptake, and heme biosynthesis.

Globin gene expression is the best characterized aspect of $\mathrm{Hb}$ formation. It has been studied intensively as a

Correspondence: Nancy C. Andrews M.D., Ph.D., Division of Hematology, Enders 720, Children's Hospital, 300 Longwood Ave., Boston, MA 02115.

Supported by a Merck-AFCR Foundation M.D.-Ph.D. postdoctoral fellow award, a Leukemia Society of America Special Fellow award, and a Hood Foundation Child Health Research Grant.

${ }^{1}$ Recipient of the Society for Pediatric Research 1994 Young Investigator Award for Research in Pediatrics. model system for understanding tissue-specific gene control. Humans have two types of $\alpha$-globin chains and four types of $\beta$-globin chains that can form heterotetramers. During embryonic life, $\mathrm{Hb}$ consists of $\zeta_{2}-\epsilon_{2}$ tetramers. The composition of $\mathrm{Hb}$ switches in the fetus, and $\alpha_{2}-\gamma_{2}$ tetramers predominate. Around birth the globin configuration switches again, to the adult $\alpha_{2}-\beta_{2}$ pattern.

Our current understanding of human globin gene expression is based on the work of a number of laboratories, including those of Arthur Nienhuis, George Stamatoyannopoulos, Frank Grosveld, Y. W. Kan, and Stuart Orkin (reviewed in ref. 1). Analyses in these laboratories and others have led to the understanding that globin genes are organized in developmentally regulated $\alpha$-like and $\beta$-like clusters on human chromosomes 16 and 11 (Fig. 1). The expression of each individual globin gene is controlled by its own transcriptional promoter (indicated by arrows in Fig. 1). However, activation of high-level transcription requires additional enhancer-like DNA sequences that act on all genes of the cluster. These elements, termed locus control regions (LCR), are positioned many kilobases upstream of the globin genes. LCR are tissue specific. Although they act as extremely potent enhancers in erythroid cells, LCR do not activate transcription of the globin genes in nonerythroid tissues.

Close examination of the structure of the LCR gives clues to their potency and tissue specificity. LCR are marked by segments of exposed chromatin, which are hypersensitive to digestion by deoxyribonuclease in an in vitro assay. Zooming in on the DNA sequences found

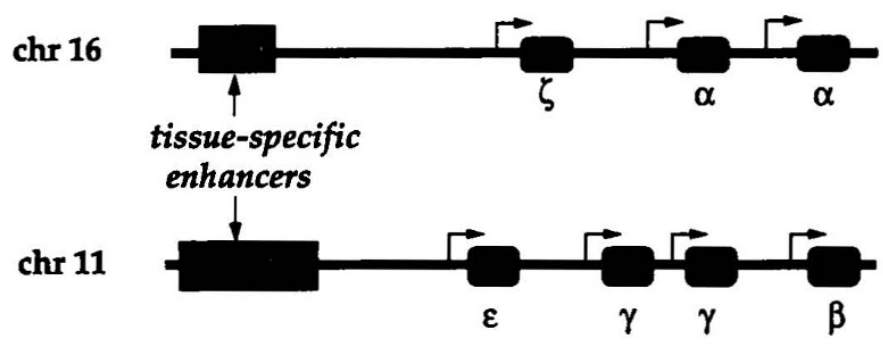

Figure 1. Organization of the human $\alpha$ - and $\beta$-globin gene clusters. A simplified cartoon illustrates the configuration of genes encoding $\alpha$-like and $\beta$-like globin proteins, located on chromosomes (chr) 16 and 11, respectively. Arrows indicate individual gene promoters. LCR are located far upstream of the gene clusters and serve as tissue-specific enhancers. 
within these hypersensitive sites, we find arrays of three types of sequence motifs, which can bind transcriptional regulatory factors (Fig. 2). CACC motifs bind factors expressed in all cells, and they will not be discussed in detail. Of greater interest are the GATA motifs and NF-E2 motifs, which are recognized by factors found only in hematopoietic cells. GATA motifs bind the abundant transcription factor GATA-1.

GATA-1 was cloned and characterized in the laboratories of Stuart Orkin and Gary Felsenfeld $(2,3)$. It is a hematopoiesis-specific member of the zinc finger class of DNA-binding transcription factors. Gene targeting experiments, in which the GATA-1 gene is destroyed in the mouse germline, have shown that GATA-1 is globally important for red cell development (4). GATA-1 binds to regulatory sequences in the promoters and enhancers of virtually all erythroid genes.

The last type of LCR sequence motif binds another erythroid-specific transcription factor, designated NFE2. NF-E2 was originally identified by Paul-Henri Romeo and colleagues as a DNA-binding activity that recognizes a complex, 11-bp motif (5). Embedded within the NF-E2 binding site is a 7-bp sequence that is recognized by widely expressed AP-1 transcription factors. AP-1 factors include jun and fos proteins, which play important roles in regulation of expression of a wide variety of eukaryotic genes. As detailed below, the binding site similarity is not simply a coincidence-NF-E2 turns out to be a specialized member of a large family of AP-1-like factors. We became particularly interested in NF-E2 because several groups had reported that NF-E2 seemed to be a primary mediator of the potent erythroidspecific globin enhancer activity of the LCR (6-8).

Parallel studies in France and Japan suggested another role for NF-E2 in regulating the expression of heme biosynthetic enzymes. Control of heme biosynthesis is remarkably complex, involving multiple enzymatic steps. Heme synthesis begins and ends in the mitochondrion, but intermediate reactions are carried out in the cytoplasm. All cells must produce heme for cytochromes, but red cells must produce much more heme for $\mathrm{Hb}$. Details of the expression of heme biosynthetic enzyme genes are

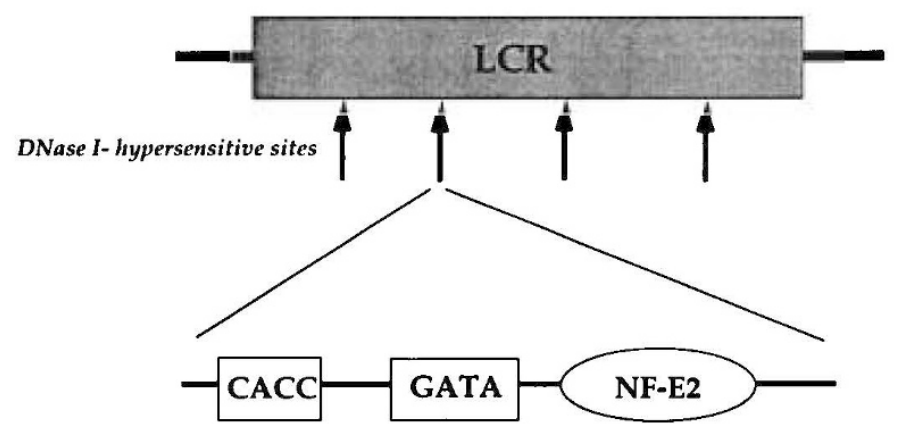

Figure 2. LCR are marked by DNase I-hypersensitive sites corresponding to transcription factor-binding motifs. Examination of nucleotide sequences at DNase I-hypersensitive sites within the globin LCR reveals recognition motifs for general transcription factors (CACC sites) and erythroid-restricted factors GATA-1 and NF-E2. still being worked out, but many seem to be differentially regulated in erythroid and nonerythroid cells.

Transcription factor NF-E2 appears to be important for red cell expression of at least two key heme biosynthetic enzymes, porphobilinogen deaminase and ferrochelatase $(5,9)$. Both of these enzymes are strategically positioned in the heme biosynthetic pathway, porphobilinogen deaminase at an early step, and ferrochelatase at the final step in which iron is inserted into the newly formed protoporphyrin molecule to make heme. Indirectly, this suggests that NF-E2 is also an important regulator of heme biosynthesis.

Molecular cloning seemed critical for understanding the role of NF-E2 in control of erythroid gene expression. To this end, we purified NF-E2 DNA-binding activity from mouse erythroleukemia or MEL cells (10-12). We worked out an in vitro DNA-binding assay for NF-E2 to follow activity through purification. Extracts were prepared from the nuclei of $700 \mathrm{~g}$ of MEL cells and fractionated by heparin-sepharose chromatography and DNA affinity chromatography. The final yield was about $1 \mu \mathrm{g}$ of protein. SDS-polyacrylamide gel analysis demonstrated two major polypeptide species copurifying with NF-E2 activity with apparent molecular masses of $18 \mathrm{kD}$ and 45 $\mathrm{kD}$. These molecules were designated $\mathrm{p} 45$ and $\mathrm{p} 18$. We predicted that both proteins would be essential components of NF-E2, because NF-E2 has an apparent molecular mass of about $60 \mathrm{kD}$, consistent with the sum of one 45-kD subunit and one 18-kD subunit.

In collaboration with Drs. Hediye Erdjument-Bromage and Paul Tempst, we obtained partial amino acid sequence of both polypeptides. Using the genetic code, we designed degenerate oligonucleotides that would correspond to all possible combinations of codons that might encode the fragments of determined amino acid sequence. These degenerate oligonucleotides were used for library screening and the polymerase chain reaction, and a combination of these methods allowed us to isolate cDNA clones encoding both p45 and p18 (10-12).

Sequence analysis revealed that both clones encode novel proteins, which belong to the AP-1 superfamily of transcriptional regulators. 45 contains 373 amino acids, and $\mathrm{p} 18$ contains 156 amino acids. Each protein has its own basic region-leucine zipper domain resembling homologous domains in other AP-1-like proteins. The basic regions specify DNA binding, with their positively charged amino acids contacting negatively charged DNA. The leucine zippers form $\alpha$-helical structures with hydrophobic surfaces, which act like Velcro to promote protein-protein dimerization.

Although neither $\mathrm{p} 45$ nor $\mathrm{p} 18$ had been described previously, each was related to several other basic-zipper proteins. About $40 \%$ of the sequence of $\mathrm{p} 45$, including the basic region and the leucine zipper, is homologous to a Drosophila protein called cnc (13). Cnc is a developmentally regulated gene product that is believed to contribute to formation of head and neck structures in the fly. Two other proteins-NRF from human cells (14) and skn from 
Caenorhabditis elegans (15)-share this extensive homology with $c n c$. Together these proteins compose a new cnc-like subfamily of basic-leucine zipper molecules.

Similarly, p18 is highly related to a chicken oncogene, v-maf (16), its cellular counterparts, chicken proteins maf $f$ and maf $k$, and a human retina-specific protein, NRL (17). These proteins comprise a maf-like subfamily, with homology in the basic-zipper domain and in several other regions.

It appeared that $\mathrm{p} 45$ and $\mathrm{p} 18$ formed a heterodimeric protein, together composing the NF-E2 transcription factor. To prove this, and to further characterize NF-E2, we did additional experiments, which I will describe briefly $(10,11)$. An electrophoretic mobility shift or gel shift assay allows detection of NF-E2 DNA-binding activity in vitro. Protein extracts from cell nuclei are incubated with short, radiolabeled DNA fragments carrying the specific NF-E2 binding site. Samples are electrophoresed through a nondenaturing acrylamide gel and analyzed by autoradiography. Unbound DNA probe migrates rapidly to the bottom of the gel, whereas probe that has bound to NF-E2 protein is retarded. This allows direct visualization of the DNA-protein interaction.

To prove that both are components of native NF-E2, we prepared specific antibodies against recombinant $\mathrm{p} 45$ and p18 and tested their effects on NF-E2 DNA-binding activity in the gel shift assay (Fig. 3). Radiolabeled NF-E2 site probe was incubated with protein extract from mouse erythroleukemia cells. The band in the first lane of Figure 3, indicated by the arrow, corresponds to specific binding of NF-E2 transcription factor to its DNA recognition motif. The next few lanes show the NF-E2 binding reaction in the presence of several different rabbit sera. As shown in the second and fourth lanes, preimmune sera had no effect on NF-E2 binding. In contrast, as shown in the third lane, antiserum prepared against p45 caused aggregation of the NF-E2 DNA-protein complex, preventing it from entering the gel. A weaker antiserum prepared against p18 also disrupted NF-E2 binding, causing a portion of the complex to be held up higher in the gel, resulting in the supershift shown in the last lane. These results indicated that both $\mathrm{p} 45$ and $\mathrm{p} 18$ are components of NF-E2, consistent with our hypothesis that native NF-E2 is a p45/p18 heterodimer.

Figure 4 shows a cartoon of the structure of NF-E2 bound to DNA. p18 and p45 dimerize through their leucine zippers and together determine DNA-binding specificity. p18 recognizes $7 \mathrm{bp}$ of the binding site, and p45 recognizes the remaining $4 \mathrm{bp}$. This complex likely interacts with other cellular factors to result in transcriptional activation.

NF-E2 DNA-binding activity had been found only in extracts from erythroid and megakaryocytic cells. We predicted that $\mathrm{p} 45, \mathrm{p} 18$, or both proteins would have a similar tissue distribution, to account for NF-E2 specificity. Northern blots were used to analyze the expression patterns of mRNA encoding these proteins. p45 mRNA was detected in a subset of hematopoietic cells

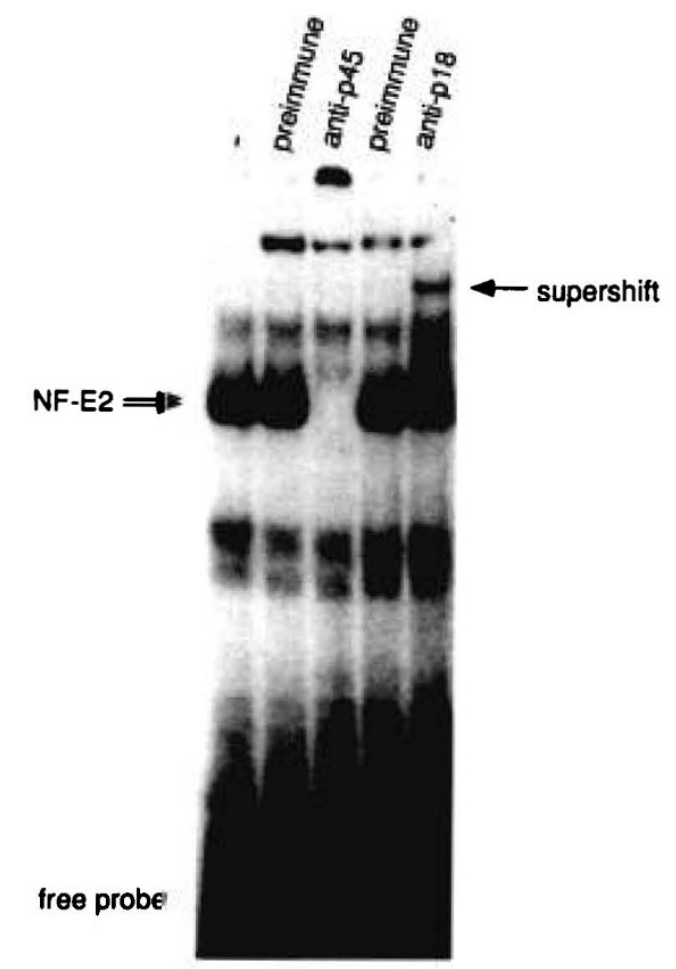

Figure 3. Anti-p45 and anti-p18 antibodies disrupt NF-E2 proteinDNA interactions in vitro. A gel shift experiment is shown; the experimental procedure is described in the text. The NF-E2 DNA-protein complex is indicated by double arrows. Added antisera are identified at the top of each lane. Anti-p45 antiserum completely disrupts the NF-E2 complex; anti-p18 antiserum supershifts a portion of it.

and in hematopoietic organs, but it was not seen in a variety of other tissues. Surprisingly, however, p45 mRNA was detected at low levels in the small intestine. In contrast, $\mathrm{p} 18$ is ubiquitously expressed. Thus the $\mathrm{p} 45$ component accounts for the tissue pattern of NF-E2 DNA-binding activity.

We took advantage of the fact that $\mathrm{p} 45$ is not expressed in COS monkey kidney cells to reconstitute native NF-E2 in vivo. We introduced expression constructs containing p45 and p18 cDNA into COS cells by transfection and compared DNA-binding activity in nuclear extracts with that found in MEL cells (Fig. 5). The MEL cell control pattern is shown in the first lane of Figure 5. The COS

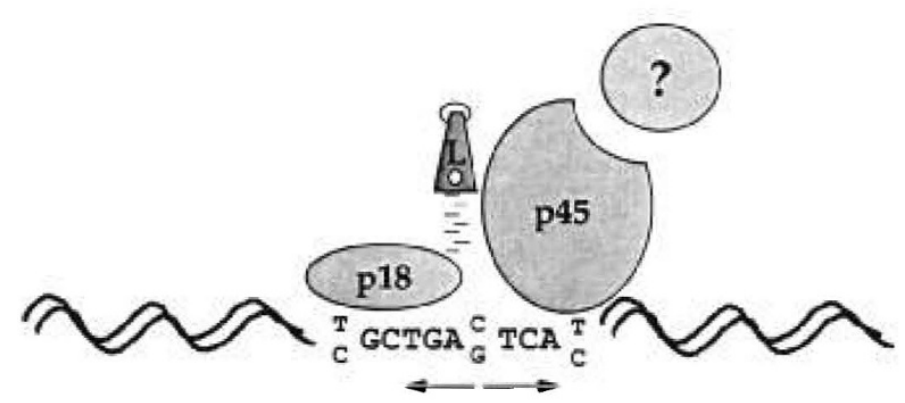

Figure 4. Subunit structure of native NF-E2. The cartoon shows our working model of transcription factor NF-E2. p18 and p45 subunits dimerize through leucine zipper interactions and bind an 11-bp recognition sequence. Additional factors, as yet uncharacterized, likely interact with p45 to mediate transcriptional activation. 
transfectant lanes all show endogenous AP-1-binding activity, producing DNA-protein complexes that migrate more slowly than NF-E2 complexes. In the first transfectant lane, we find that introduction of p45 cDNA alone generates NF-E2 DNA-binding activity that comigrates with NF-E2 naturally found in mouse erythroleukemia cells. This must result from dimerization of expressed p45 protein with p18 protein endogenous to COS cells. When p45 and p18 cDNA were coexpressed, as in the second transfectant lane, the amount of NF-E2 activity generated was much greater. This suggests that $\mathrm{p} 18$ is limiting when p45 cDNA is expressed alone. Finally, expression of p18 alone, in the last lane, produces no NF-E2 activity, because the $\mathrm{p} 45$ protein is not normally present in COS cells. Additional assays showed that the recombinant NF-E2 expressed in COS cells is indistinguishable from native erythroid NF-E2 and that it can activate transcription of a reporter gene linked to NF-E2binding sites.

The chromosomal localization of the p45 NF-E2 gene in the mouse was determined in collaboration with Drs. Luanne Peters, Eva Eicher, and Sam Lux. Intriguingly, the p45 gene maps to mouse chromosome 15, colocalizing with a previously mapped mouse phenotype termed microcytic anemia and abbreviated $m k$ (18). In the 1960s, Elizabeth Russell and coworkers reported that homozygous $m k / m k$ mutant mice have severe hypochromic, microcytic anemia with abnormal intestinal iron uptake and defective red cell iron utilization (reviewed in ref. 19). Elegant genetic and bone marrow transplantation experiments showed that the $m k$ defect is intrinsic to both red cells and intestinal cells.

Although NF-E2 had not been suspected to play any role in iron uptake or metabolism, it was intriguing that the $\mathbf{p} 45$ gene mapped in the vicinity of a locus associated with anemia. We hypothesized that the $m k$ phenotype might be due to a mutation within the p45 NF-E2 gene. There were several lines of evidence that supported our

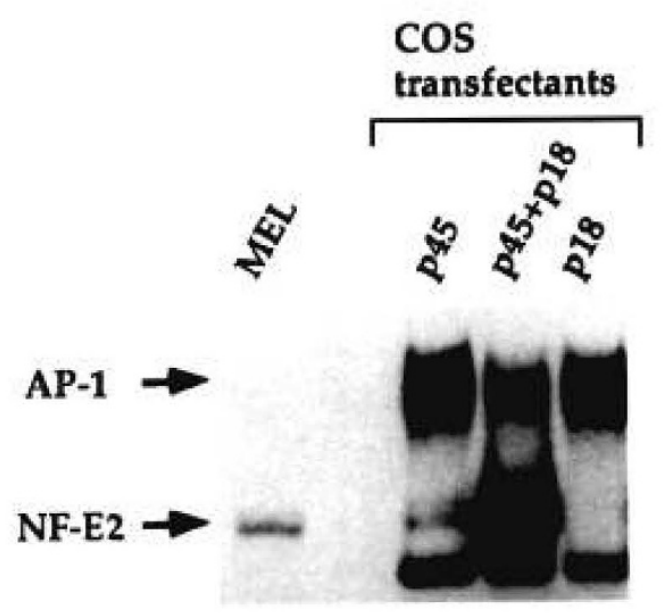

Figure 5. Coexpression of cDNA encoding p45 and p18 generates NF-E2 activity in COS cells. This gel shift experiment is described in the text. NF-E2 and AP-1 DNA-protein complexes are indicated by arrows. Cell sources of nuclear extracts are identified at the top. hypothesis (18). First, p45 appears to be expressed exclusively in those tissues that are affected in $m k$ mice: hematopoietic cells and small intestine. Second, there is a single nucleotide difference in the $\mathrm{p} 45$ gene found in $m k$ mice compared with DNA from the parental strain on which the $m k$ mutation arose. Third, the $m k$ phenotype of hypochromic, microcytic anemia is consistent with predicted effects of a mutation in p45 NF-E2. And finally, transfection of expressible NF-E2 cDNA into COS cells stimulates those cells to take up iron (unpublished data). Taken together, these data strongly suggested that NF-E2 might regulate the expression of one or more iron transport proteins, supporting the hypothesis that the $m k$ mutation resides within the p45 gene (18). However, we have recently determined that, although NF-E2 does appear to be involved in regulation of iron utilization, it is not the protein defective in $\mathrm{mk} / \mathrm{mk}$ mice (unpublished data). That protein must be closely linked to p45, and it awaits molecular characterization.

When we began this work, we knew that NF-E2 was implicated in the regulation of expression of globin genes and two or more heme biosynthetic enzyme genes. Our recent data suggest that NF-E2 also regulates iron uptake and utilization. Thus, this single heterodimeric transcription factor, NF-E2, appears to be a master coordinator of all three aspects of $\mathrm{Hb}$ synthesis. We believe that NF-E2, acting in both red cells and intestine, is involved in transcriptional regulation of production of globin chains, biosynthesis of heme, and uptake of iron. Teleologically, it makes sense that this sort of regulator should exist, to allow enormous amounts of $\mathrm{Hb}$ to be produced without accumulating toxic unused components.

Much remains to be learned about how this is accomplished and how NF-E2 itself is regulated. We have focused our future investigations on the least understood aspect of $\mathrm{Hb}$ formation, the uptake and utilization of elemental iron. We are now searching for the protein affected in $m k$ mutant mice to better understand iron uptake in the intestine and iron unloading in erythroid cells.

Acknowledgments. This work was carried out in large part while the author was a postdoctoral fellow in Stuart Orkin's laboratory. Paul Tempst and Hediye ErdjumentBromage carried out amino acid sequence analysis from purified protein. Paul Ney and Karen Kotkow assisted in isolating the cDNA clone encoding p18. Luanne Peters, Eva Eicher, and Sam Lux determined the chromosomal map position of the $\mathrm{p} 45$ gene in the mouse.

\section{REFERENCES}

1. Orkin SH 1990 Globin gene regulation and switching: circa 1990. Cell 63:665672

2. Tsai S-F, Martin DIK, Zon LI, D’Andrea AD, Wong GG, Orkin SH 1989 Cloning of cDNA for the major DNA-binding protein of the erythroid lineage through expression in mammalian cells. Nature 339:446-451

3. Evans T, Felsenfeld G 1989 The erythrocyte-specific transcription factor Eryfl: a new finger protein. Cell 58:877-885 
4. Pevny L, Simon MC, Robertson E, Klein WH, Tsai S-F, D'Agati V, Orkin SH 1991 Erythroid differentiation in chimaeric mice blocked by a targeted mutation in the gene for transcription factor GATA-1. Nature 349:257-260

5. Mignotte V, Wall L, deBoer E, Grosveld F, Romeo P-H 1989 Two tissuespecific factors bind the erythroid promoter of the human porphobilinogen deaminase gene. Nucleic Acids Res 17:37-54

6. Talbot D, Philipsen S, Fraser P, Grosveld F 1990 Detailed analysis of the site 3 region of the human beta-globin dominant control region. EMBO J 9:2169-2178

7. Talbot D, Grosveld F 1991 The 5' HS2 of the globin locus control region enhances transcription through the interaction of a multimeric complex bind ing at two functionally distinct NF-E2 binding sites. EMBO J 10:1391-1398

8. Ney PA, Sorrentino BP, Lowrey CH, Nienhuis AW 1990 Inducibility of the HSII enhancer depends on binding of an erythroid specific nuclear protein. Nucleic Acids Res 18:6011-6017

9. Taketani S, Inazawa J, Nakahashi Y, Abe T, Tokunaga R 1992 Structure of the human ferrochelatase gene. Exon/intron gene organization and location of the gene to chromosome 18. Eur J Biochem 205:217-222

10. Andrews NC, Erdjument-Bromage H, Davidson M, Tempst P, Orkin SH 1993 Erythroid transcription factor NF-E2 is a haematopoietic-specific basicleucine zipper protein. Nature 362:722-728

11. Andrews NC, Kotkow KJ, Ney PA, Erdjument-Bromage H, Tempst P, Orkin SH 1993 The ubiquitous subunit of erythroid transcription factor NF-E2 is a small basic-leucine zipper protein related to the v-maf oncogene. Proc Natl Acad Sci USA 90:11488-11492

12. Ney PA, Andrews NC, Jane SM, Safer B, Purucker ME, Morton CC, Weremowicz S, Goff SC, Orkin SH, Nienhuis AW 1993 Purification of the human NF-E2 complex: cDNA cloning of the hematopoietic cellspecific subunit and evidence for an associated partner. Mol Cell Biol 13:5604-5612

13. Mohler J, Vani K, Leung S, Epstein A 1991 Segmentally restricted, cephalic expression of a leucine zipper gene during Drosophila embryogenesis. Mech Dev 34:3-10

14. Chan JY, Han X-L, Kan YW 1993 Cloning of Nrf1, an NF-E2-related transcription factor, by genetic selection in yeast. Proc Natl Acad Sci USA 90:11371-11375

15. Bowerman B, Eaton BA, Priess JR 1992 Skn-1, a maternally expressed gene required to specify the fate of ventral blastomeres in the early $C$. elegans embryo. Cell 68:1061-1075

16. Nishizawa M, Kataoka K, Goto N, Fujiwara KT, Kawai S 1989 V-maf, a viral oncogene that encodes a "leucine zipper" motif. Proc Natl Acad Sci USA 86:7711-7715

17. Swaroop A, Xu J, Pawar H, Jackson A, Skolnick C, Agarwal N 1992 A conserved retina-specific gene encodes a basic motif/leucine zipper domain. Proc Natl Acad Sci USA 89:266-270

18. Peters LL, Andrews NC, Eicher EM, Davidson MB, Orkin SH, Lux SE 1993 Mouse microcytic anaemia caused by a defect in the gene encoding the globin enhancer-binding protein NF-E2. Nature 362:768-770

19. Russell ES, Nash DJ, Bernstein SE, Kent EL, McFarland EC, Matthews SM, Norwood MS 1970 Characterization and genetic studies of microcytic anemia in house mouse. Blood 35:838-850 